\title{
Latino parents' perceptions of the eating and physical activity experiences of their pre-school children at home and at family child-care homes: a qualitative study
}

\author{
Ana C Lindsay $1,2, *$, Mary L Greaney ${ }^{3}$, Sherrie F Wallington ${ }^{4}$, Faith D Sands ${ }^{5}$, \\ Julie A Wright ${ }^{1}$ and Judith Salkeld ${ }^{2}$ \\ ${ }^{1}$ Exercise and Health Sciences Department, University of Massachusetts Boston, College of Nursing and Health \\ Sciences, 100 Morrissey Boulevard, Boston, MA 02 125, USA: ${ }^{2}$ Department of Nutrition, Harvard School of \\ Public Health, Boston, MA, USA: ${ }^{3}$ Health Studies \& Department of Kinesiology, University of Rhode Island, Kingston, \\ RI, USA: ${ }^{4}$ Lombardi Comprehensive Cancer Center, Georgetown University, Washington, DC, USA: ${ }^{5}$ Program in \\ Gerontology, University of Rhode Island, Kingston, RI, USA
}

Submitted 12 January 2016: Final revision received 22 June 2016: Accepted 29 June 2016: First published online 19 August 2016

\begin{abstract}
Objective: Research indicates that healthful eating and physical activity (PA) practices implemented in child-care settings can have a positive effect on children's healthful behaviours in this setting, and this effect on healthful behaviours may possibly transfer to the home environment. While more research is needed to examine whether behaviours learned in family child-care homes (FCCH) transfer, the potential for transferability is especially important given that Latino children's home environment has been characterized by obesogenic parenting practices. We aimed to examine Latino parents' perceptions of their preschool children's eating and PA experiences at home and at FCCH.

Design: Qualitative study. Six focus groups were conducted in Spanish ( $n$ 36). Transcripts were analysed using thematic analysis to identify key concepts and themes. Results: Analyses revealed that Latino parents perceive their children have healthier eating and PA experiences at FCCH than at home. Parents attributed this to FCCH providers providing an environment conducive to healthful eating and PA due to providers having more knowledge and skills, time and resources, and being required to follow rules and regulations set by the state that promote healthful eating and PA.

Conclusions: Understanding parental perceptions, attitudes and practices related to establishing and maintaining an environment conducive to children's healthful eating and PA at home and at the FCCH is essential for the design of successful interventions to promote children's healthful behaviours in these two settings. Given that parents perceive their children as having more healthful behaviours while at FCCH, interventions that address both settings jointly may be most effective than those addressing only one environment by itself.
\end{abstract}

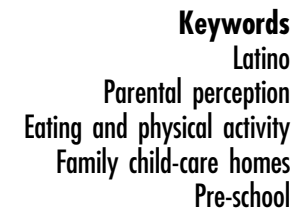

eywords

eption Eating and physical activity Pre-school
Latinos are the largest and most rapidly growing population group in the USA ${ }^{(1)}$ and children in low-income Latino families are at elevated risk of becoming overweight and obese, making childhood obesity among Latinos a pressing public health concern because childhood weight status tracks into adulthood ${ }^{(2)}$. Racial/ethnic disparities in being overweight and obese among minority and immigrant children in the USA, including Latinos, are associated with socio-economic disparities (e.g. lower maternal education and family income $)^{(3-5)}$. A recent study by Guerrero et $a l .{ }^{(5)}$ using a nationally representative cohort of children of pre-school age found that significant racial and ethnic differences exist in early childhood BMI trajectories, with African-American and Latino children displaying higher BMI growth trajectories than white children. Furthermore, Latino children from primarily Spanish-speaking households had the highest mean BMI trajectories ${ }^{(5)}$. Substantive efforts are needed to prevent obesity among Latino children if future trends in chronic diseases are to be altered in this population ${ }^{(2)}$.

The home and child-care settings are important early social environments for the development and maintenance of children's early dietary and physical activity (PA) habits ${ }^{(6-12)}$. Parents and caregivers are influential in children 
developing healthful behaviours during early childhood, which is when diet and PA habits are formed ${ }^{(6,8)}$.

Early education and care (EEC) programmes are important settings for preventing obesity ${ }^{(7-9)}$ and for developing healthful eating and PA behaviours in young children ${ }^{(7-9)}$. EEC programmes are growing in importance due to parents' increasing reliance on child care for their young children $^{(13-15)}$. Almost two-thirds of pre-school children attend EEC programmes, such as Head Start, child-care centres and family child-care homes $(\mathrm{FCCH})^{(8)}$. More than 1.9 million pre-school children attend FCCH, and this EEC setting is the second largest provider of non-relative care in the USA for children up to 5 years old ${ }^{(11,12)}$. Many Latino families prefer FCCH to other EEC settings due to cultural preferences for family-like care, flexible hours and lower costs, thus making FCCH an ideal setting for obesity prevention targeting Latino families and children ${ }^{(16)}$.

Research indicates that healthful eating and PA practices implemented in child-care settings can positively affect children's healthful behaviours in this setting ${ }^{(17-20)}$. Moreover, this effect on healthful behaviours may possibly transfer to the home environment ${ }^{(21,22)}$. A recent qualitative study by Mena et al. ${ }^{(22)}$ revealed that Latino parents believe having their children attend a child-care centre with healthful practices will influence their family choices and home environment. While more research is needed to examine whether behaviours learned in FCCH transfer to the home and family, the potential for transferability is especially important given that Latino children's home environment is often characterized by obesogenic parenting practices ${ }^{(23)}$.

Although a growing number of studies have focused on FCCH as a setting for promoting early healthful eating and $\mathrm{PA}^{(17-20,24-26)}$, to our knowledge no study has examined Latino parents' perceptions of their children's eating and PA experiences in FCCH run by Latino providers. Therefore, the purpose of the current qualitative study was to examine Latino parents' perceptions of their children's eating and PA experiences while at home and at FCCH. Understanding parents' perceptions and attitudes is essential to the development and implementation of culturally and family-appropriate interventions that account for the day-to-day context of parents and families ${ }^{(27,28)}$.

The Social Ecological Model (SEM) was used to construct the moderator guide for the focus group discussions. The SEM is often utilized to explore personal, family, environmental and organizational influences on parents' beliefs, attitudes and practices amenable to intervention and modification ${ }^{(29)}$.

\section{Methods}

\section{Study design}

The current study was part of a multicomponent qualitative research project exploring factors influencing eating, PA and sedentary behaviours among Latino children of pre-school age attending FCCH in Massachusetts, USA ${ }^{(20)}$.
We worked with the Massachusetts EEC and Child Care Circuit to enrol FCCH, utilizing master lists of all licensed Latino FCCH providers in the state, and randomly selected twenty-five providers in each area of the state (North Shore, Greater Boston, Central MA and Western MA). We mailed each selected provider a flyer in Spanish with a telephone number to call for additional information. Interested providers were screened for eligibility (Latino, having at least three children aged $2-5$ years in the FCCH). Eligible providers who enrolled in the study ( $n$ 45) assisted our research staff in recruiting Latino parents with children between 2 and 5 years of age attending their FCCH to participate in the current study.

\section{Study participants}

A total of six focus groups (four to seven participants per group) with thirty-six Latino parents or primary caregivers (twenty-seven mothers, six fathers, three grandmothers) of pre-school children (2-5 years), representing thirty-six unique families, were conducted before thematic saturation was reached. Participants were originally from South America (e.g. Colombia, Peru), Central America (e.g. Guatemala, Honduras, El Salvador), Mexico or the Caribbean (e.g. Dominican Republic, Puerto Rico), and had been in the USA for an average of 9 years. Parents' mean age was 29.0 years, and grandmothers' mean age was 58.7 years. Approximately $61 \%$ had not completed high school, and all participants predominantly spoke Spanish at home. Participants had an average of two children (range: 1-4) per household and reported that their children spent on average $8.5 \mathrm{~h}$ (range: 6-11 h) per day in the FCCH (see Table 1).

\section{Procedures}

A native Spanish-speaker trained in qualitative research methods moderated the focus groups in Spanish using a discussion guide with open-ended questions and probes (see Table 2). The pilot-tested guide explored: (i) parents' beliefs and attitudes related to eating and PA; (ii) parents' perceptions of children's eating and PA experiences at home; (iii) parenting practices related to eating and PA behaviours at home; (iv) barriers faced in structuring a home environment conducive to healthful eating and PA; (v) parents' perceptions of children's eating and PA experiences at $\mathrm{FCCH}$; and (vi) communication between parents and $\mathrm{FCCH}$ providers. The same focus group discussion guide was used for all focus groups.

The study was approved by the Institutional Review Board for the Protection of Human Subjects at Harvard T.H. Chan School of Public Health. Focus groups, held in meeting rooms of public libraries between April and September 2015, lasted approximately $90 \mathrm{~min}$ and were audio-taped after participants provided signed informed consent.

Before each group, the moderator explained procedures and participants completed a brief, self-administered questionnaire assessing education, marital status, country of origin 
Table 1 Sociodemographic and acculturation characteristics of focus group participants: Latino parents $(n$ 36) of preschool children, Massachusetts, USA

\begin{tabular}{|c|c|c|c|c|}
\hline Characteristic & Mean & SD & $n$ & $\%$ \\
\hline Age (years) ${ }^{*}$ & 29.0 & $2 \cdot 3$ & & \\
\hline Hispanic or Latino & & & 36 & $100 \cdot 0$ \\
\hline Foreign-born & & & 34 & 94.4 \\
\hline \multicolumn{5}{|l|}{ Country of origin } \\
\hline Colombia & & & 9 & $25 \cdot 0$ \\
\hline Dominican Republic & & & 9 & $25 \cdot 0$ \\
\hline Guatemala & & & 5 & $14 \cdot 0$ \\
\hline Puerto Rico & & & 4 & 11.0 \\
\hline Peru & & & 3 & 8.4 \\
\hline USA & & & 2 & $5 \cdot 6$ \\
\hline Mexico & & & 2 & $5 \cdot 6$ \\
\hline El Salvador & & & 1 & $2 \cdot 7$ \\
\hline Honduras & & & 1 & $2 \cdot 7$ \\
\hline Years in the USA & 9.0 & $2 \cdot 4$ & & \\
\hline \multicolumn{5}{|l|}{ Predominant language spoken at home } \\
\hline Spanish & & & 36 & $100 \cdot 0$ \\
\hline \multirow{2}{*}{\multicolumn{5}{|c|}{ Marital status }} \\
\hline & & & & \\
\hline Married & & & 24 & 66.7 \\
\hline Divorced/separated & & & 8 & $22 \cdot 1$ \\
\hline Widowed & & & 2 & $5 \cdot 6$ \\
\hline Single & & & 2 & $5 \cdot 6$ \\
\hline Number of children & $2 \cdot 2$ & $\begin{array}{c}1.4 \\
\text { (range: } 1-4 \text { ) }\end{array}$ & & \\
\hline \multicolumn{5}{|l|}{ Education level } \\
\hline Less than high school & & & 22 & $61 \cdot 1$ \\
\hline High school degree & & & 8 & $22 \cdot 2$ \\
\hline General Education Diploma & & & 5 & 14.0 \\
\hline Missing & & & 1 & 2.7 \\
\hline \multicolumn{5}{|l|}{ Annual household annual income (\$US) } \\
\hline$>20000-<40000$ & & & 23 & 63.9 \\
\hline$<20000$ & & & 13 & $36 \cdot 1$ \\
\hline Average time child spends in family child-care home $(\mathrm{h} / \mathrm{d})$ & 8.5 & $\begin{array}{c}3.0 \\
\text { (range 6-11) }\end{array}$ & & \\
\hline
\end{tabular}

*Average age of parents was calculated for both mothers $(n 27)$ and fathers $(n 6)$ participating in the focus groups; average age of the grandmothers $(n 3)$ participating in the focus groups was 58.7 years.

and length of time living in the USA. At the start of the focus group, the moderator directed participants to think about their oldest pre-school child (2-5 years) when participating in the discussion. Participants received a \$US 25 gift card.

\section{Analyses}

Audio tapes were transcribed verbatim in Spanish and translated into English without identifiers. To ensure that the integrity and equivalence of the data was not lost in the process of translation, a professional transcriptionist, bilingual and native Spanish-speaker translated the transcripts using forward-backward techniques to establish semantic equivalence in translation.

Transcripts were analysed using thematic analysis ${ }^{(30)}$, an iterative process of coding data in phases to create meaningful patterns ${ }^{(30-34)}$, using the SEM as a framework. Analytic phases included data familiarization, generation of initial codes, searching for and review of themes and patterns, and defining and naming themes ${ }^{(31,33)}$. Two authors, both experienced qualitative researchers, independently conducted all analyses, checked for consistency between their analyses and resolved discrepancies. During the coding process and data analyses, the two authors responsible for coding met regularly and read the codes and focus group reports independently ${ }^{(30-32)}$. The reliability of coding was determined by measuring the ratio of number of agreements against the sum of agreements plus disagreements ${ }^{(30,32-34)}$. In most cases, the inter-coder reliability was over $85 \%$. Disagreements were discussed until a consensus was reached. Descriptive statistics were calculated for the sociodemographic data using Microsoft Excel $2008^{\circledR}$.

\section{Results}

Emergent themes related to parents' and grandparents' (hereafter referred to as 'parents') perceptions of their children's eating and PA experiences at FCCH and at home are discussed below. Identified themes are categorized using the SEM when applicable.

\section{Themes related to children's eating and physical activity experiences at bome}

Individual level

Parents are aware of the importance of healthful eating and physical activity. There was consensus among 
Table 2 Questions from the focus group discussion guide on Latino parents' perceptions of children's eating and physical activity (PA) experiences at home and in family child-care homes (FCCH), Massachusetts, USA

Topic
Parents' perceptions of children's eating and PA at home
Parents' beliefs and attitudes related to healthy eating

Parents' perceptions of children's eating and feeding experiences at home

Parenting practices related to a child's eating and feeding at home

Parents' perceptions of children's PA at home

Parenting practices related to screen time at home

Barriers faced by parents in structuring a home environment conducive to healthful eating and PA
Discussion guide questions

Describe what healthy eating means to you. (Probes: How would you describe a 'healthy meal'? How do you decide if a food is good for health? What foods do you think are good for health?)

Describe how satisfied you are with the types and amounts of food your child eats at home. (Probes: Why? Is it the same as what the rest of family eats? Is it culturally appropriate? Is it healthy?)

Describe some foods that you wish your child would eat more of or more often. (Probes: Why?)

Describe what types of foods you wish your child would eat less of or less often. (Probes: Why?)

Describe what your biggest concerns are about how your child eats. (Probes: Do you ever worry your child does not eat enough? Do you ever worry that your child eats too much? Do you ever worry about not having enough food to provide to your child?)

Describe a typical mealtime routine at your home. (Probes: Who regularly prepares the meal? Who is present at the meal? Regularity of mealtime: Do you usually sit together as a family to eat? Does your child eat separately from the rest of the family? Does your child eat the same type of food as the rest of the family? Is the TV usually on during mealtimes?)

Describe how you decide what foods to feed your child. (Probes: Healthy foods; cost/convenience; cultural values and traditions; according to advice or direction from another person? If yes, probe further: What is that person's role or relationship to you/your child?)

Describe how you decide how much food to feed your child. (Probes: Based on child's age? Based on what you think is the 'right' amount? Based on what child eats and on how much on a regular basis?)

Describe what happens if your child does not eat as much as you think he or she should eat. (Probes: Do you verbally convince your child that he or she should eat more? Do you physically force your child to eat more? For example, by spoon-feeding her? Do you try to negotiate with your child or offer him/her a reward such as a dessert or a toy for eating?)

Describe any limits to eating that you set for your child during meals. (Probes: How about snacks? Any particular types of foods and/or drinks not allowed?) Describe how important you think it is for children to be physically active.

(Probes: Do you think it is a problem for kids to spend too much time being sedentary or not being physically active?)

Describe how much PA you think children need on a daily basis. (Probes: Why?)

Describe how your child is physically active at home. (Probes: Plays actively outside? Plays actively inside? Does not play actively when at home?)

Describe how satisfied you are with the amount of PA your child engages in while at home. (Probes: Why? Do you wish he/she would be more active at home? Do you wish he/she had more outdoor playtime when at home?)

Describe some types of PA or active play your child engages in while at home. (Probes: Riding his/her bike? Playing in nearby playground or park?)

Describe some of your biggest concerns about how physically active your child is. (Probes: Do you ever worry your child does not engage in enough PA? Do you ever worry that your child is too sedentary?)

Describe what types of things would you like to change about how physically active your child is at home. (Probes: Amount? Types of activity?)

Describe rules that you have at home regarding your child watching TV and/or videos or playing with electronics. (Probes: Time limit on TV and electronics? Not allowing the TV on during meals? Not allowing TV during the week?)

Describe any electronic equipment your child owns (e.g. TV, computer or portable video game). (Probes: Do you let your child keep these electronics at all times? Are these kept in your child's easy reach?)

Describe barriers that you face in making sure that your child eats or drinks what you think is 'good' or 'healthy' for him or her at home. (Probes: Time? Cost? Knowledge of what and how much to feed your child? Influence of other people in the household? (e.g. older siblings, grandparents, father, mother))

Describe barriers that you face in making sure that your child is physically active when at home. (Probes: Space? Time? Household obligations?

Neighbourhood safety? Knowledge of how physically active your child should be? Influence of other people in the household? (e.g. older siblings, grandparents, father, mother))

Describe barriers that you face in making sure that your child does not spend too much time sedentary time such as watching TV and/or videos or playing video games at home. (Probes: Lack of household rules? Space? Time? Other obligations that keep you from having time to take your child out to play?) 
Table 2 Continued

Topic Discussion guide questions

Parents' perceptions of children's eating and PA experiences in $\mathrm{FCCH}$

Parents' perceptions of children's eating and feeding Describe how satisfied you are with the types and amount of food your child eats experiences at $\mathrm{FCCH}$ in the FCCH. (Probes: Why? Is it healthy? Is it of good variety? Is it culturally appropriate?)

Describe what types of and how much food and drink your child eats and drinks at the FCCH. (Probes: How do you know? Does it ever change?)

Describe how similar or different the food that your child eats in the $\mathrm{FCCH}$ is from the food that you serve at home. (Probes: Types of foods and drinks served? Serving size? Time offered? Snacks? Cultural foods?)

Describe what meals your child eats at the FCCH. (Probes: All meals? (breakfast, lunch, snacks, supper) Every day? Some days?)

Describe what meals you send with your child to eat at the FCCH. (Probes: Breakfast, lunch and/or supper, special snacks? Special drinks/beverages?)

Parents' perceptions of children's PA and screen time experiences at $\mathrm{FCCH}$

Describe how satisfied you are with your child's levels of PA while he/she is at the FCCH. (Probes: Amount? Types of activity?)

Describe in what ways is your child physically active while at the $\mathrm{FCCH}$. (Probes: Types of activities, equipment, duration?)

Describe rules or practices your family child-care provider has related to children being physically active while at the FCCH. (Probes: What sorts of rules? Does she set a good example for children being active?)

Describe things that make it easy or hard for children to be active at the $\mathrm{FCCH}$. (Probes: Space? Equipment? Neighbourhood?)

Describe anything else you would like to change about how much and/or what types of activity your child does at the $\mathrm{FCCH}$. (Probes: Amount of time he or she is active? Why?)

Describe rules or policies your family child-care provider has related to screen time for children while at the FCCH. (Probes: Amount? Types?)

Describe how comfortable you are with the amount of TV your child watches at the FCCH. (Probes: Why?)

Describe rules or policies that your family child-care provider has about children bringing any electronics to the family child care. (Probes: Never? Only on special occasions?)

Describe how satisfied you are with how much screen time your child is allowed to have while at the FCCH. (Probes: Why? Amount? Time?)

Communication between parents and $\mathrm{FCCH}$ providers Describe how you learn about your child's daily activities (e.g. eating, playing, sleeping) at his/her FCCH. (Probes: How do you know what your child ate during the day at the FCCH? How about his/her physical activities?)

Describe ways that your family child-care provider communicates with you about your child's day at the FCCH in general. (Probes: How often? What types? (pamphlets, newsletters))

Describe any other ways that your family child-care provider shares information about your child's nutrition and PA while he/she is at the FCCH. (Probes: Or in general?)

Describe any additional information about your child's eating and PA you would like to get from the family child-care provider.

Describe how you would like to get information about your child during the time he/she spends at the FCCH. (Probes: Printed information? Text? Email? Phone call?)

Final remarks

Is there anything else you would like to share with us related to your children's eating and PA experiences at home or at the $\mathrm{FCCH}$ ?

TV, television.

parents about the importance of children eating healthfully and being physically active, and most recognized the importance of developing these habits early in life. As one mother stated:

'I think children eating a healthy diet and being active is really important, and they have to learn and start having these habits when they are little, so that they get used to eating and grow healthy.'

Nevertheless, several parents acknowledged that despite their intentions and efforts, their children do not always eat healthy foods and may not be physically active at home. One parent mentioned:

'As parents, we know it is important for children to eat healthy and be physically active, but it is not always as easy as it sounds making sure that children want to eat healthy foods, and making sure that you have time to take them out to play.'

Parents believe that children's eating habits at bome need to change. Most parents stated they would like their children to eat more healthfully at home, including 
eating more fruits and vegetables and less sweets. One parent said:

'I like for her [daughter] to eat more vegetables. She doesn't like vegetables. She says I have to add some dressing so she can eat the vegetables ... she doesn't like the taste.'

\section{Interpersonal and socio-cultural context level}

Parents are important role models. Parents spoke of their own eating behaviours influencing those of their children, but acknowledged that being a positive role model was challenging. One parent mentioned:

'We all need to role model good eating habits for our children ... we should show portion control as adults and also not eat what children leave on their plates.'

Similarly, another parent said:

'It is not always easy to do what you know is right ... you are tired and still have a lot to do, so it's easy to go for fast food and just think that it's only one day ... but I think it is important to try.'

Parents also recognized their role in instilling PA habits for their children. As one parent said:

'I try to show my son that it's important to be physically active. We play soccer together, and I encourage him to play in our local soccer league.'

Competing demands make it difficult to have family eating routines. Most parents reported that lack of time due to competing daily demands (e.g. multiple household chores, work obligations, long workdays) make it difficult to eat together as a family and establish set eating routines such as family dinners during the week. A parent said:

'During the week, I [am] busy with work and have less time, so usually the kids eat first. I try to prepare something quick, give them a bath and put them in bed.'

Several parents reported they are more likely to eat together as a family on weekends. As a parent mentioned:

'During the weekends, I try to make some time for cooking, and on Sundays, especially, we usually eat together as a family. That's when we have more time.'

Additionally, several parents mentioned that it is difficult to establish eating routines during the week as they are often tired from working long hours and do not have the energy to 'enforce' eating rules and routines for their children. One parent said:

'In my house, there are no set rules. I buy whatever food the kids like. If my daughter doesn't want vegetables, then I tell her, "Well don't eat them."
I come home and I am tired, the kids are also tired. It is really hard for all of us after a long day of work ...'

A few parents also reported that they did not want to disagree with their children about what they should eat after spending many hours apart. For example, one parent reported:

'We spend many hours apart, and most days of the week ... I don't think it's good to be "fighting" about food and what she [daughter] should eat or not eat ... so I just don't.'

Nevertheless, some parents spoke of their efforts to enforce family eating routines because they view this as their responsibility. A parent reported:

'I try to plan some meals in advance for the whole week so I can try and have them eat healthier foods ... I feel it's my job as they will eat what I serve.'

Latino culture positively influences children's and families' eating habits. Parents spoke of their Latino culture positively influencing their children's and family's eating habits. One parent mentioned:

'I feel like my culture plays a role in what I buy to eat. For example, in Colombia we eat lots of vegetables and hearty soups, and I try to keep that as part of our family traditions.'

Furthermore, parents spoke of their culture as having a positive influence on their children's and family's choice of foods. One parent said:

'I love to eat my traditional foods and the vegetables from my country. And it also makes me happy to cook and see my children eating some of the foods I grew up eating.'

Spending time outdoors and being physically active are important aspects of Latino culture. Parents discussed that central aspects of Latino culture and traditions are spending time outdoors and being physically active. Nevertheless, nearly all acknowledged that their daily lives in the USA do not allow for them and their families to be as physically active as they were in their home countries. As one parent stated:

'Here, you are always busy with work, trying to take care of your family, and there is not much time left to go out, be physically active, and relax. It's a different pace of life.'

Another parent added:

'I grew up outside, running, playing soccer, being very physically active. My children don't spend much time outside; they are either in school, daycare, or inside the house. During the summer is when we spend more time outside, but it's only a few months.' 
Physical activity is important, but not a family priority. Nearly all parents spoke of PA as being 'important for our children'; however, they acknowledged that their children are not always physically active. As one parent explained:

'I think it's really important for kids to be physically active, but there are days that [it] is really hard to get them out to play and spend some energy.'

Although some parents spoke of trying to ensure that their children are physically active when home, several reported being 'too busy' to do so. One parent said:

'My son likes to play baseball and my husband tries to play with him. If it is left to me, I don't have time. ... It's really hard, you want them to be active but you have to find the time, and it's not easy.'

Parents face barriers to belping their children be active at home. The majority of parents spoke of barriers such as lack of time, lack of space, safety concerns and cold weather that prevented their children from physically active while at home. As one parent stated:

'By the time you get home and have to get dinner ready, there isn't much time left to take the kids outside to play. They end up sitting and watching some TV while I get things done.'

Another parent explained:

'I don't feel safe letting the kids go outside and play by themselves. An adult needs to be with them at all times. The streets are not safe.'

\section{Home environment level}

Strategies parents use to promote bealthful eating at home. Several parents discussed trying to incorporate a variety of strategies to influence healthful eating behaviours of their children, such as controlling portion size and not having 'junk' food at home. One parent said:

'I try to not buy or bring junk food into the house. If we have cookies and sweets in the house, we all eat.

So, I just try not to have those foods in the house.'

Excess screen time at bome. When discussing how children spend time at home, almost all parents said that their children engage in excessive screen time. Parents stated that their children have more screen time (e.g. television viewing and playing video games) at home than at FCCH. One parent said:

'At home my daughter watches a lot more TV than at daycare. The first thing she does when she gets home is to watch TV...'

Furthermore, several parents said that the television is always on when they were home, including during meals; even when no one is watching a programme, the television is on in the background.

\section{Themes related to parents' perceptions of children's eating and physical activity experiences at family child-care bomes}

Individual level

Children engage in healthier behaviours at family childcare bomes than at home. Nearly all parents expressed the belief that their children eat more healthfully at FCCH than at home. For example, one parent stated:

'My daughter eats all kinds of fruits and vegetables served at her daycare, but at home she always rejects when I tell her that she should eat her vegetables ... She just doesn't eat at home the foods that she eats at the home daycare.'

Some parents noted that children learned to eat what they are served, including fruits and vegetables, while at the $\mathrm{FCCH}$ due to the FCCH having set menus. One parent reported:

'My son has really learned to eat his fruits and vegetables at the daycare. He knows he needs to eat what is served. He sees the other kids eating, and that helps him try.'

Another parent explained:

'I try to set rules at home, but the reality is that my daughter eats healthier foods at the daycare. At home she eats foods that children are not allowed to have at the home daycare like sweets.'

Another parent added:

'At my daughter's daycare, the provider has rules of what children are and are not allowed to eat. Besides, she [provider] has time to prepare the foods, and does [so] in a way that the kids think the food is actually good. My daughter would never eat broccoli at home, but at the family child care, she says it is [broccoli] like eating the trees in the forest. They have songs they sing when they eat, they count the colours of the different foods ... I don't have time or ideas for any of that.'

Furthermore, parents spoke of their children spending more time being physically active and less time in sedentary activities at FCCH than at home. They felt that the structured routine of $\mathrm{FCCH}$ that incorporated time for children to be physically active resulted in their children being more active at $\mathrm{FCCH}$ than at home. As one parent mentioned:

'The child-care provider takes the children out to play every day. Even when it is cold, she says that children need to go out and get some physical activity and fresh air.'

Interpersonal level

Family child-care home providers have the ability to promote bealthful behaviours. Overwhelmingly, parents 
reported that their $\mathrm{FCCH}$ providers have the knowledge, skills and time to provide healthful meals and snacks and to ensure their children are physically active while at $\mathrm{FCCH}$. As one parent stated:

'My child-care provider is very good - she makes sure that the kids eat healthy, are active, and have time to play.'

Furthermore, some parents believed that FCCH providers had more resources to plan and serve healthful meals than they had. One parent explained:

'My child-care provider is very well informed about good nutrition for young children. She is always giving me information that she gets from workshops and conferences that she goes to. It's a requirement for her job. I trust she is doing the best for my child ... and my daughter eats more healthy foods when she is at the daycare than when she is at home.'

\section{Environmental and organizational levels}

Parents are supportive of foods served at family child-care homes. Most parents reported being very satisfied with the types of foods their children were served at FCCH and believed their children are learning healthful eating habits. Overwhelmingly, as discussed earlier, parents reported that their children ate more healthfully and consumed a greater variety of healthy foods at the FCCH than at home. As one parent reported:

'At daycare they tend to be healthier [meals] because they are stricter with food. They also serve a healthy variety of foods. At home, I have more [unhealthy] options. So, by far, my son eats better at the daycare.'

Family child-care bome licensing requirements promote physical activity and limit screen time. Parents perceived that FCCH licensing requirements promote healthful PA behaviours among their children and, as a result, children are more active when in this setting. As one parent explained:

'At the daycare they take her out, there is more physical activity. They have to go out, it's a requirement. When I get home, there is no time - I have to fix the house, prepare them something to eat, bathe them, etcetera. So, there is not a lot of time. So, I am glad my son can play and be active when he is at daycare.'

Moreover, parents reported that children watch less television and play fewer video games while at FCCH than at home.

Parents also viewed FCCH providers as being more skilled than they are at managing screen time rules and routines. As one parent said:

'In her daycare they watch cartoons or movies but for only about $15-30 \mathrm{~min}$ while the provider prepares their food. I know that on weekends they watch more television with me.'

Another parent added:

'I believe that there should be daycare on weekends! In her daycare, they have strict rules about TV. Also, at my house there are no rules. They do what they want. That is why I wish there was daycare on weekends. They would feed my kids correctly, monitor their screen time, and return them to me at 5.'

\section{Policy-related factors}

State rules and regulations promote bealthful eating and physical activity PA in family child-care homes. Parents spoke of their child-care providers having to meet training requirements and being required to follow nutrition and PA regulations set by state agencies, which results in them being more knowledgeable than parents are about nutrition and healthy eating. As one parent explained:

'My child-care provider follows a plan. They are better informed on the right kinds of food to serve the children. They have to follow and serve healthy foods to the children ... they are required to take courses, which is really good for the care they provide to the children.'

Parents also noted that the state regulations impact PA and screen behaviours. One parent stated:

'At daycare, the children are not allowed to watch a lot of TV, the child-care provider has activities for them to do when they come in, and they are only allowed to watch $30 \mathrm{~min}$ of TV after lunch when she is cleaning up and getting the children ready for naptime.'

Another parent noted:

'My provider tells me that the state agency does not allow them to let kids watch a lot of TV. She [provider] doesn't even let the kids bring any electronic devices to the daycare. She [provider] is required to make sure that the kids go out and play and are physically active. She needs to follow the rules.'

\section{Discussion}

The purpose of the current study was to examine Latino parents' perceptions of their children's eating and PA experiences at home and at FCCH. This information is essential for the development and implementation of interventions that are compatible with parents' and families' perceptions, attitudes and practices of children's eating and PA behaviours ${ }^{(27,28,35,36)}$. Analyses determined that parents perceived that their children have healthier eating and PA behaviours at FCCH than at home. Parents attributed this to FCCH providers being more knowledgeable, having more 
skills, resources and time than parents do, and being required to follow rules and regulations set by the state that promote healthful eating and PA.

Moreover, an important theme identified in our study was parents' trust in FCCH providers to promote healthful eating and PA for their children. This finding may be particularly important for low-income, ethnic minority parents, who may benefit from having a person of similar culture provide information, education and modelling on how to structure and reinforce culturally sensitive healthy eating and PA for children ${ }^{(7,17,18,25,26)}$. Latino parents' trust in their Latino FCCH providers suggests that providers may be well positioned to engage and help parents establish home environments conducive to children's development of healthful eating and PA behaviours. FCCH providers could provide parents with important skill-building materials and training for effective parenting, such as limit setting. FCCH providers could disseminate individualized information about the child's day (e.g. what the child ate and amount of PA) and facilitate access for parents to evidence-based information that is linguistically and culturally appropriate through in-person individual and group meetings, bulletin boards or e-communications. Moreover, FCCH providers' health promotion efforts could extend beyond the children and the families they serve since they are often members of the same communities and culture of these children and families, making them a natural and sustainable part of the daily social context.

Our findings also highlight the importance of understanding and addressing the social day-to-day context of Latino families when designing interventions to promote healthy eating and PA among young children. Interventions that do not address the context of Latino families will likely not be successful. Parents in our study discussed daily life demands that impacted their ability to create and maintain a home environment conducive to healthful eating and PA habits. This finding is important because previous studies indicate that children living in households with regular family routines (e.g. regularly eating meals as a family) are at lower risk of obesity than those who do not have household routines ${ }^{(10-12)}$.

Although it is essential that parents recognize the importance of healthful behaviours, parents need to have the self-efficacy to make changes to the home and family environment that support children's healthy eating and $\mathrm{PA}^{(27,28)}$. Most parents participating in our study recognized the importance of healthful eating and PA, but appeared to lack the confidence to make the changes needed to create a home environment supportive of these behaviours. Future studies should explore parental selfefficacy and readiness to make changes supportive of healthful eating and PA behaviours for their children and family.

Study results should be considered in the light of some limitations, including limited generalizability due to purposive sampling and a relatively small sample of low-income, diverse Latino parents in four areas of Massachusetts. Because the purpose of the study was to explore parents' perceptions and attitudes about their children's eating and PA environments at home and in $\mathrm{FCCH}$, the findings cannot be used to extrapolate children's actual dietary intake or PA levels at home and at FCCH. Future studies using objective assessments (e.g. accelerometers and direct observation of foods consumed) are necessary to describe children's behaviour. Additionally, parents who chose to participate in the study could have a heightened interest and awareness regarding the focus group topics. Future research can address these limitations by exploring influences on Latino parents' beliefs, attitudes and practices from other communities across the USA and employing mixed methods to strengthen the validity of the findings. Nevertheless, the study's qualitative methodology provided deeper insight into personal perceptions and experiences of Latino parents concerning their children's eating and PA experiences at home and at FCCH. Finally, while a strength of the study was its use of the SEM, the outer levels (organizational and policy) need to be explored further, perhaps including key informants from these levels ${ }^{(27,28)}$.

\section{Conclusions}

Understanding parental perceptions, attitudes and practices related to establishing and maintaining an environment conducive to children's healthful eating and PA at home and at FCCH is essential for the design of successful interventions to promote children's healthful behaviours in these two settings. Study findings revealed that parents view multiple factors, including individual, interpersonal, social context, home environment, organizational and policy factors, as influencing pre-school children's healthy eating and PA experiences at home and at FCCH. Therefore, interventions targeting the home and FCCH settings serving Latino children should address multiple levels of the SEM to create healthful eating and PA experiences. Addressing both the home and FCCH settings will likely increase the impact of designed interventions. Furthermore, given that parents perceive their children as having more healthful behaviours while at $\mathrm{FCCH}$, interventions that address both settings jointly may be most effective than those addressing only one environment by itself $^{(27,28,37)}$.

\section{Acknowledgements}

Acknowledgements: The authors are grateful for the assistance of personnel at the Massachusetts Department of Early Education and Care, Child Care Circuit, Family Care Systems, and Child and Adult Care Food Program, 
especially Yours for Children, Inc. The authors are thankful to the FCCH providers and parents who participated in this study. Financial support: This study was supported by Aetna Foundation Inc. (grant number 11-02395; A.C.L., Principal Investigator). Conflict of interest: The authors declare that there is no conflict of interest regarding the publication of this paper. Authorship: The co-authors contributed to the work as follows: A.C.L. in study design, data collection, data analysis and manuscript preparation and review; M.L.G. in study design, manuscript preparation and manuscript review; S.F.W. in development of the theoretical framework for the study, manuscript preparation and manuscript review; F.D.S. in data analysis and manuscript review; J.A.W. in manuscript preparation and manuscript review; and J.S. in data collection, data analysis and manuscript review. All authors read and approved the final manuscript. Ethics of buman subject participation: This study was approved by the Institutional Review Board for the Protection of Human Subjects at the Harvard T.H. Chan School of Public Health. Written informed consent was obtained from all participants.

\section{References}

1. Ogden CL, Carroll MD \& Flegal KM (2014) Prevalence of obesity in the United States. JAMA 312, 189-190.

2. Nyberg K, Ramirez A \& Gallion K (2011) Physical activity, overweight and obesity among Latino youth. Salud America! http://www.rwif.org/en/library/research/2011/12/ physical-activity--overweight-and-obesity-among-latino-youth. html (accessed August 2016).

3. Rossen LM (2014) Neighbourhood economic deprivation explains racial/ethnic disparities in overweight and obesity among children and adolescents in the USA. $J$ Epidemiol Community Health 68, 123-129.

4. Rossen LM \& Talih M (2014) Social determinants of disparities in weight among US children and adolescents. Ann Epidemiol 24, 705.e2-713.e2.

5. Guerrero AD, Mao C, Fuller B et al. (2016) Racial and ethnic disparities in early childhood obesity: growth trajectories in body mass index. J Racial Ethn Health Disparities 3, 129-137.

6. Spurrier NJ, Magarey AA, Golley R et al. (2008) Relationships between the home environment and physical activity and dietary patterns of preschool children: a crosssectional study. Int J Behav Nutr Phys Act 30, 31.

7. Larson N, Ward DS, Neelon SB et al. (2011) What role can child-care settings play in obesity prevention? A review of the evidence and call for research efforts. J Am Diet Assoc 111, 1343-1362.

8. Lindsay AC, Sussner KM, Kim J et al. (2006) The role of parents in preventing childhood obesity. Future Child $\mathbf{1 6}$ 169-186.

9. Story M, Kaphingst KM \& French S (2006) The role of child care settings in obesity prevention. Future Child 16, $143-168$.

10. Anderson SE \& Whitaker RC (2010) Household routines and obesity in US preschool-aged children. Pediatrics $\mathbf{1 2 5}$, 420-428.

11. Cespedes EM, McDonald J, Haines J et al. (2013) Obesityrelated behaviors of US- and non-US-born parents and children in low-income households. J Dev Behav Pediatr 34, 541-548.
12. Jones BL \& Fiese BH, STRONG Kids Team (2014) Parent routines, child routines, and family demographics associated with obesity in parents and preschool-aged children. Front Psychol 29, 374.

13. Centers for Disease Control and Prevention (2011) Early Child Care and Education (ECE). Atlanta, GA: CDC; available at https://www.cdc.gov/obesity/strategies/ childcareece.html

14. Laughlin L (2013) Who's Minding the Kids? Child Care Arrangements: Spring 2011. Household Economic Studies Report no. P70-135. Washington, DC: US Department of Commerce, US Census Bureau; available at http://www. census.gov/content/dam/Census/library/publications/2013/ demo/p70-135.pdf

15. Forum on Child and Family Statistics (2009) Forum on Child and Family Statistics America's Children: Key National Indicators of Well-Being. Washington, DC: US Government Printing Office.

16. Daugherty L (2010) Child care choices of Hispanic families: why aren't families using child care? Doctoral dissertation, Pardee Rand Graduate School; available at http://www. rand.org/pubs/rgs_dissertations/RGSD258.html

17. Brann LS (2010) Child-feeding practices and child overweight perceptions of family day care providers caring for preschool-aged children. J Pediatr Health Care 24, 312-317.

18. DeSilva-Sanigorski A, Elea D, Bell C et al. (2011) Obesity prevention in the family day care setting: impact of the Romp \& Chomp intervention on opportunities for children's physical activity and healthy eating. Child Care Health Dev 37, 385-393.

19. Santiago-Torres M, Adams AK, Carrel AL et al. (2014) Home food availability, parental dietary intake, and familial eating habits influence the diet quality of urban Hispanic children. Child Obes 10, 408-415.

20. Kipping R, Jago R, Metcalfe C et al. (2016) NAP SACC UK: protocol for a feasibility cluster randomised controlled trial in nurseries and at home to increase physical activity and healthy eating in children aged 2-4 years. BMJ Open 6, e010622.

21. Gunter KB, Rice KR, Ward DS et al. (2012) Factors associated with physical activity in children attending family child care homes. Prev Med 54, 131-133.

22. Mena NZ, Gorman K, Dickin K et al. (2015) Contextual and cultural influences on parental feeding practices and involvement in child care centers among Hispanic parents. Child Obes 11, 347-354.

23. Lindsay AC, Salkeld JA \& Greaney ML (2015) Latino family childcare providers' beliefs, attitudes, and practices related to promotion of healthy behaviors among preschool children: a qualitative study. J Obes 2015, 409742.

24. Trost SG, Messner L, Fitzgerald K et al. (2011) A nutrition and physical activity intervention for family child care homes. Am J Prev Med 41, 392-398.

25. Østbye T, Mann CM, Vaughn AE et al. (2015) The keys to healthy family child care homes intervention: study design and rationale. Contemp Clin Trials 40, 80-89.

26. Rosenthal MS, Crowley AA \& Curry L (2013) Family child care providers' self-perceived role in obesity prevention: working with children, parents, and external influences. J Nutr Educ Behav 45, 595-601.

27. Bluford DA, Sherry B \& Scanlon KS (2007) Interventions to prevent or treat obesity in preschool children: a review of evaluated programs. Obesity (Silver Spring) 15, 1356-1372.

28. Skouteris H, McCabe M, Swinburn B et al. (2010) Parental influence and obesity prevention in pre-schoolers: a systematic review of interventions. Obes Rev 12, 315-328.

29. McLeroy KR, Bibeau D, Steckler A et al. (1988) An ecological perspective on health promotion programs. Health Educ Q 15, 351-377. 
30. Vaismoradi M, Turunen H \& Bondas $\mathrm{T}$ (2013) Content analysis and thematic analysis: implications for conducting a qualitative descriptive study. Nurs Health Sci 15, 398-405.

31. Silverman D (2006) Interpreting Qualitative Data: Methods for Analysing Talk, Text and Interaction, 3rd ed. London: SAGE Publications Ltd.

32. Pope C, Ziebland S \& Mays N (1999) Analysing qualitative data. In Qualitative Research in Health Care, 2nd ed. pp. 75-88 [C Pope and N Mays, editors]. London: BMJ Books.

33. Ritchie J, Spencer L \& O'Connor W (2004) Carrying out qualitative analysis. In Qualitative Research Practice, pp. 219-262 [J Ritchie and J Lewis, editors]. London: SAGE Publications Ltd.
34. Miles M \& Huberman A (1994) Qualitative Data Analysis, 2nd ed. Thousand Oaks, CA: SAGE Publications, Inc.

35. Falbe J, Cadiz AA, Tantoco NK et al. (2015) Active and healthy families: a randomized controlled trial of a culturally tailored obesity intervention for Latino children. Acad Pediatr 15, 386-395.

36. Bender MS, Nader PR, Kennedy C et al. (2013) A culturally appropriate intervention to improve health behaviors in Hispanic mother-child dyads. Child Obes 9, 157-163.

37. Kim J, Shim JE, Wiley AR et al. (2012) Is there a difference between center and home care providers' training, perceptions, and practices related to obesity prevention? Matern Child Health J 16, 1559-1566. 